\title{
AN INTERNAL FACTOR CONTROLLING POSTERIOR REGENERATION IN SYLLID POLYCHAETES
}

\author{
By Yô K. Okada \\ Kyoto Imperial University, Japan
}

(Text-figs. I-3)

In a former paper (1929) it was stated that "regeneration takes place easily at the posterior end of divided syllids, but on cutting the most anterior part, the chitinous tube and massive proventriculus of the pharynx hinder the process of closing the wound and subsequent regeneration fails in most cases". The minimum length of so-called head pieces, in which regeneration of the tail can be effected, always comprises at least one or two more segments than those constituting the pharyngeal region. This length is internally occupied by a large chamber containing a series of highly specialized organs of ectodermal origin, extending as far as the first appearance of the moniliform intestine in a segmental cavity, which is separated from the previous one by a complete septum.

The peripharyngeal chamber is of different lengths in different species. The shortest length of the head pieces, which give caudal regeneration, also differs according to the species. The nature of this correlation between the anterior differentiation of segments and their physiological property of regeneration at the posterior end, is not yet elucidated. If, however, the cause of failure to regenerate at the posterior end in a head portion cut at the extreme anterior part of the body be assigned to the absence of certain internal structures in such an anterior portion, the formative factor of the posterior regeneration should then be correlated with the existence of such structures in the pieces and not with the physiological property of the segments involved.

Experiments with reference to this question were performed, during the summer of 1927 when I was working at the Plymouth Laboratory, on Autolytus edwarsi, which is very abundant in this district in close association with the hydroid Obelia geniculata. The anterior chamber of the species is composed of cavities corresponding to 9 or Io anterior segments and the tail regenerates only beyond the IIth setigerous segment.

In the first series of experiments the animals were cut across at different levels of the pharyngeal part within the bounds of the region presumptively non-regenerative at the posterior end, after the intestine was forced into the anterior chamber. This was done by pressing the proventriculus forward forcibly with the back of a knife. This procedure made it possible, provided 
exceedingly anterior levels were not operated on, to retain a bit of the intestine in the isolated head pieces. The latter closed the wound more easily and the specimen lived longer than in the former experiments when the cut was made simply at the corresponding levels of the body. Moreover, some pieces were detected, when observed about 2 weeks later, regenerating more or less segments at the posterior end (see Fig. I). The operation was repeated several

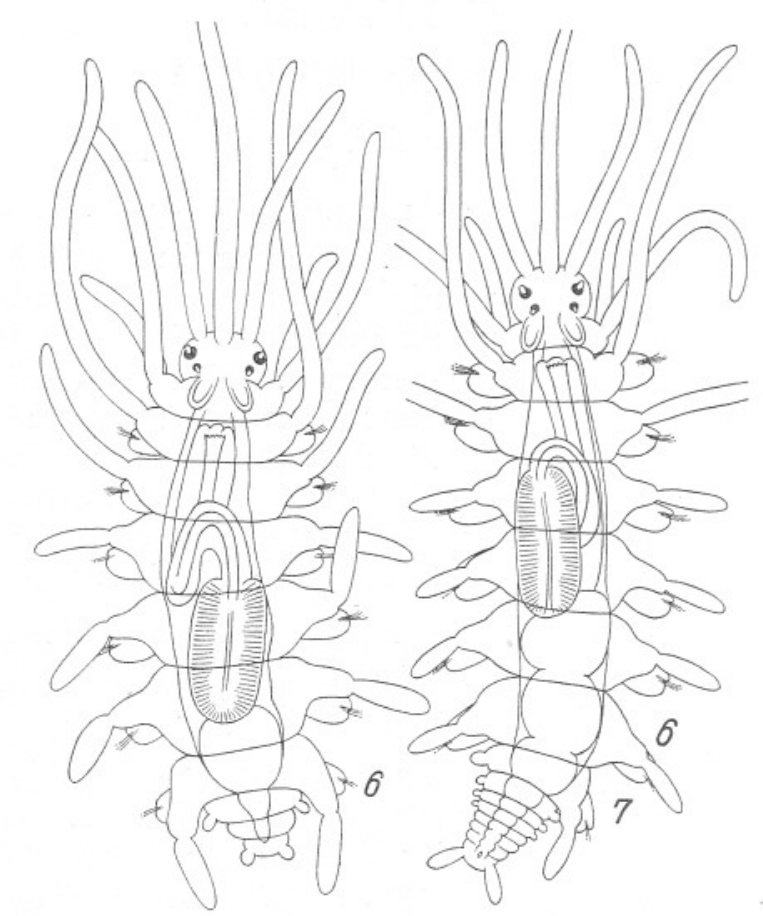

Fig. I. Tail regeneration in short head pieces of Autolytus edwarsi in the presence of the intestine.

times until a sufficient number of specimens was obtained to give conclusive results. From the results the following two facts emerged:

(I) Regeneration can take place posteriorly from any level after the 6th setigerous segment backwards, and this level at which posterior regeneration ceases is quite definite. For example, the anterior pieces isolated between the 5 th and the 6th segments do not produce a tail, but if a small fragment of the 6th segment remains attached to the posterior end of the 5th segment, regeneration occurs at this end (see Fig. 2).

(2) Where regeneration occurs, the tail does not develop beyond the state of the pygidium, unless the intestine elongates and is in close contact with the body wall at the posterior end. It is only when the last mentioned condition 


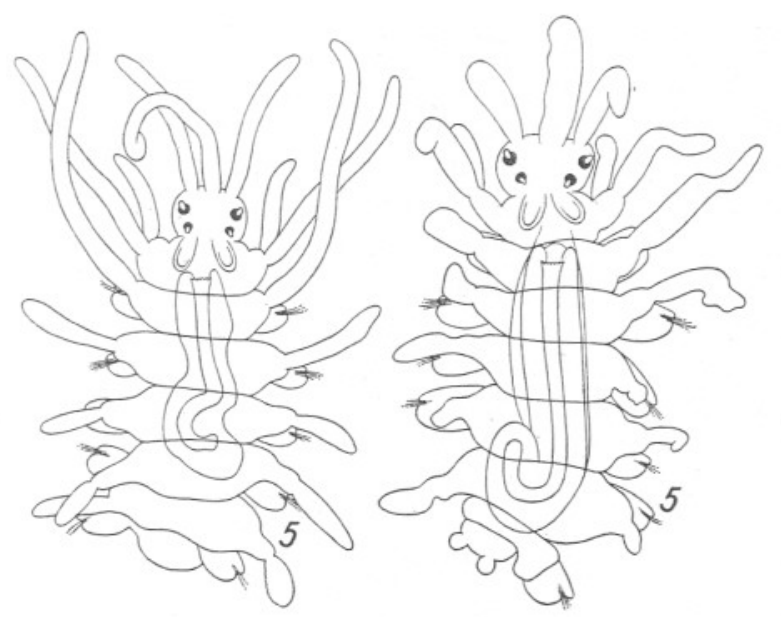

Fig. 2. Experimental demonstration of the anterior limit of tail regeneration of Autolytus edwarsi.

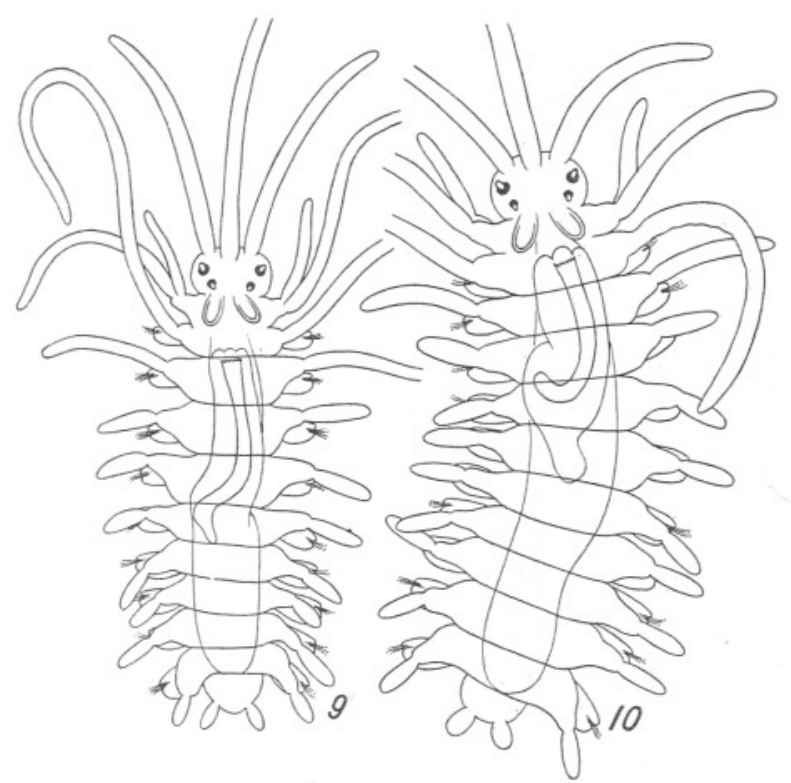

Fig. 3. Incomplete tail regeneration in long head pieces of Autolytus edwarsi in the absence of the intestine. 
is fulfilled that the development proceeds further and a long tail is produced.

That the intestine plays an important role in the caudal regeneration of the syllid is obvious. But it remains for us to show what the role is.

Thereupon the intestine was removed from fairly long anterior pieces in which caudal regeneration is normally possible. This operation was done by pressing down the proventriculus with the back of a knife and drawing it out through the wound at the posterior end by a fine hooked needle. A cut was then made in front of the pushed-down proventriculus, and the more posterior part of the alimentary canal was removed including the intestine. The animals operated on lived well as in the preceding experiment. They closed the wound and most of them regenerated a tail, which, however, did no more than develop a caudal extremity (see Fig. 3). A pair of anal cirri was quite distinct but there appeared no other segments beside the pygidium.

From the second experiment it now becomes clear that the intestine is necessary for elongation and segmentation of the new tail. The intestine itself does not seem to modify the nature of regeneration. Nevertheless, none of these regenerates without the intestine produced a heteromorphic head.

\section{REFERENCE}

OKADA, Yô K., I929. Regeneration and fragmentation in the syllidian polychaetes (Studies on the Syllidae II). Arch. Entwicklungs. Org., Bd. II5, pp. 542-600. 